\title{
A Study on the Effects of Heavy Backpack and Development of a Preventative Prototype
}

\author{
F. M. A. Hossain ${ }^{1}$ and Masuda A. Tonima ${ }^{2}$ \\ ${ }^{1}$ Stamford University Bangladesh, Dhaka, Bangladesh \\ ${ }^{2}$ BRAC University, Dhaka, Bangladesh \\ Correspondence should be addressed to F. M. A. Hossain; anim.hossain@gmail.com
}

Received 1 December 2016; Revised 13 February 2017; Accepted 19 March 2017; Published 9 May 2017

Academic Editor: Eugenio Martinelli

Copyright (c) 2017 F. M. A. Hossain and Masuda A. Tonima. This is an open access article distributed under the Creative Commons Attribution License, which permits unrestricted use, distribution, and reproduction in any medium, provided the original work is properly cited.

\begin{abstract}
Posture is a bigger concern than most think. With growing workload for school children, the weight of this issue is increasing every day. It can not only affect a child in the present but also have long-term effects on their body and render their spine vulnerable to injuries. Therefore the solutions can be either to decrease their workload, that is, decrease the weight of their backpack, or to constantly guide them to a better posture for the betterment of their spinal shape and thus their health. The aim of this paper is to find which part of the spine is affected the most by the heavy load and develop a prototype: a monitoring system that is effective in guiding a child to constantly keep their posture in check and is also simple enough to be worn every day.
\end{abstract}

\section{Introduction}

Lower back pain is an issue that most people ignore without understanding its role in one's life. This is an issue that is not restricted to any particular age group and can be caused by variety of things; it was found that people suffering from lower back pain had flatter spines as opposed to the healthier s-shape. There are many reasons for this, such as awkward back posture, manual lifting, prolonged trunk flexion, and so forth [1]; in addition to all these, aging adds further deterioration [2]. The spine changes and remodels itself depending on how one needs to change their body due to their everyday needs; hence constant guidance is needed in order to avoid remodelling and flattened spine in children, which is caused by heavy backpacks mainly [2], in order to minimize their chances of lower back pain in later life.

Studies have been conducted that confirm length of spine being affected by weight of school backpacks; with increasing weight, there were significant decreases in spine length, lumbar lordosis angle, lumbar lordosis, and sacrum inclination angle (thoracic spine length, thoracic kyphosis, and total trunk inclination were unaffected) [3].

The back posture monitoring system developed here is a simple device that is attached to a wearable belt, in which when worn the sensor detects the curvature of lumbar spine; when the user bends the sensor bends as well and after the curvature of the lumbar spine hits a threshold the system alerts the wearer of their posture condition. This system is made for the use of children and young adults; hence the device is designed to be simple and user-friendly.

\section{Literature Review}

In recent years, the weight of school backpack has become a growing concern, not just among parents but also among healthcare professionals and the school administrations, since school children have been increasingly complaining of backaches [4]. Approximately 12\% of the world population is children carrying backpacks to school every day [4]. With increasing school loads, these children are carrying backpacks heavier than $10 \%$ of their body weight on a regular basis [4]. Regardless of whether a child carries the backpack on one shoulder or two and whether the child is a male or a female, backaches were reported by all the children $[4,5]$. For understanding this issue and relationship better, all the aspects of backpack weight affecting the spine is explored in this paper. 


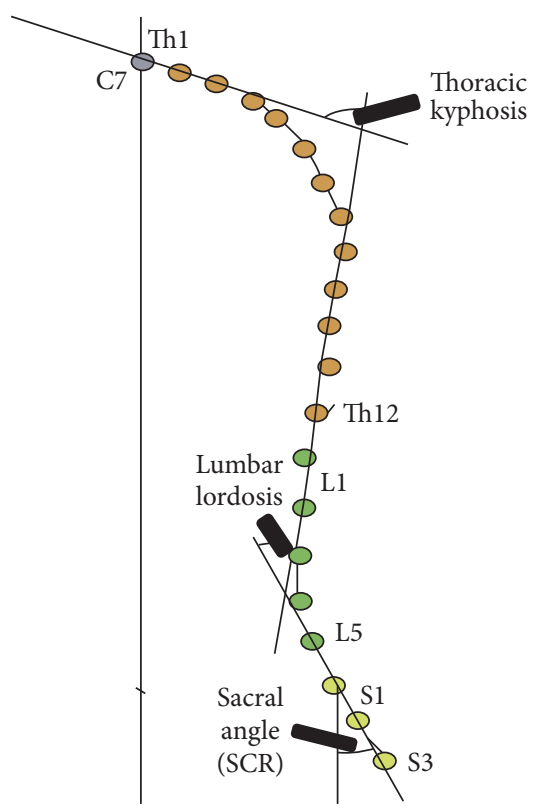

FIgURE 1: Parameters that are generally analysed of a spine [3].

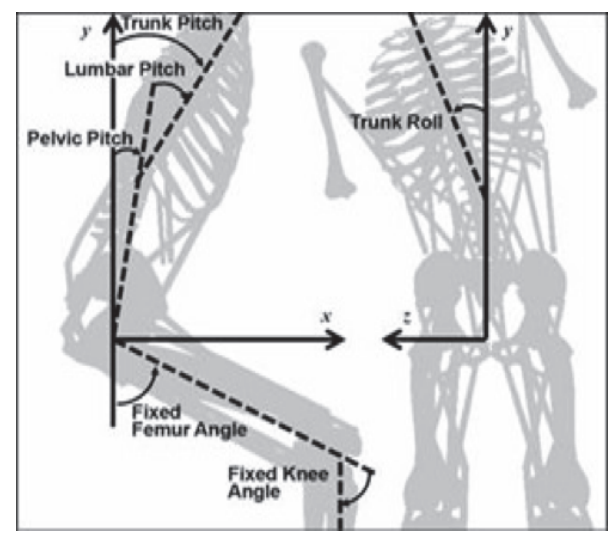

FIGURE 2: Schematic of joint angle definition that shows how trunk angle changes due to lumbar movement [6].

For complete understanding, the type of postures and understanding of how the spine works are necessary. There are four types of postures that can be identified:

(1) Thoracic kyphosis (angle)

(2) Lumbar lordosis (angle)

(3) Sacral angle

(4) Trunk angle/inclination.

Figure 1 shows the crucial parts of human spine. This can be used to relate to the aching positions in human body and for calculation in curvature of the different parts of the spine.

To understand how the trunk angle is affected, refer to Figure 2. Here it can be seen that the trunk angle is affected by multiple bends, that is, the pelvic and the lumbar.

Trunk flexion $=$ pelvic pitch + lumbar pitch .
An alternative method of calculating the trunk angle can be seen in Figure 3 . Here $\theta_{\text {Trunk }}$ can be directly measured, as shown, which is then deducted from 90 degrees to get the trunk angle. Both the methods showed in Figures 2 and 3 are effective in calculating the trunk angle.

There has been numerous studies on how daily school needs can effect a child's spine; this study explores how the thoracic, lumbar, and the trunk of the spine of a child are affected due to their daily school needs.

2.1. Thoracic. A study by Czaprowski concluded that sitting for long time, such as in schools, may contribute to longterm lower back pain. Reeducation or constant correction may prevent back pain for these kids in the future. The study conducted concerned children of age 10-14 years, where normal thoracic kyphosis for kids was measured as $42.7 \pm 9.3$ and $23.5 \pm 11.7$ in standing and sitting position, respectively, which, when the kids were asked to straighten their backs, changed significantly [8]; this study also showed that upper thoracic kyphosis only changed significantly in sitting males.

2.2. Lumbar. Study conducted in Poland showed that lumbar lordosis in females decreased significantly when they were asked to fix their posture [8]. Study conducted in Switzerland picked up changes in lumbar angles of lower back pain patients with increasing age [2]. Another study in America reports that children with heavy backpacks mostly complained about lumbar aches [4]; this study also concludes that carrying heavy backpacks leads to long-term spinal changes in children and may also cause the children to be more injury prone specifically in the affected regions [4].

2.3. Trunk. A study was conducted in India, where the subjects (200 of them) were all male children of age 12-13 and were given $5 \%$ increment of backpack weight to find the relation of weight to trunk angle deviation of these children. Study conducted in Poland found that children are more prone to tilting their torso forward in order to compensate for heavy weight of backpack, especially when backpack weight exceeds $15 \%$ of a child's bodyweight [3].

Table 1 describes the effect of backpack weight (in terms of body weight percentage) on children's spinal sections.

Table 2 helps in identifying which spinal section is most affected by the backpack's weight and in some cases even shows the action of the child while carrying the backpack. Figure 4 shows the curvilinear pattern that was observed when the children were given backpacks with percentage of their body weight during walking [11]. The three different colours represent 3 different weights of the backpack relative to the children's own bodyweight. The purple line shows how a child's posture changes over a period of time while walking with no load; in this case the child's forward lean decreases after a while. Whereas for increased weight, that is, the green and the red line, the child's forward lean increases. Since the heavy load of the backpack derails the child's spine from its normal reaction over time, it can be deduced that heavy load, as mentioned by [4], causes spinal changes in children.

Korovessis et al. concluded in one of their studies that lower back pain is caused in children only when certain 


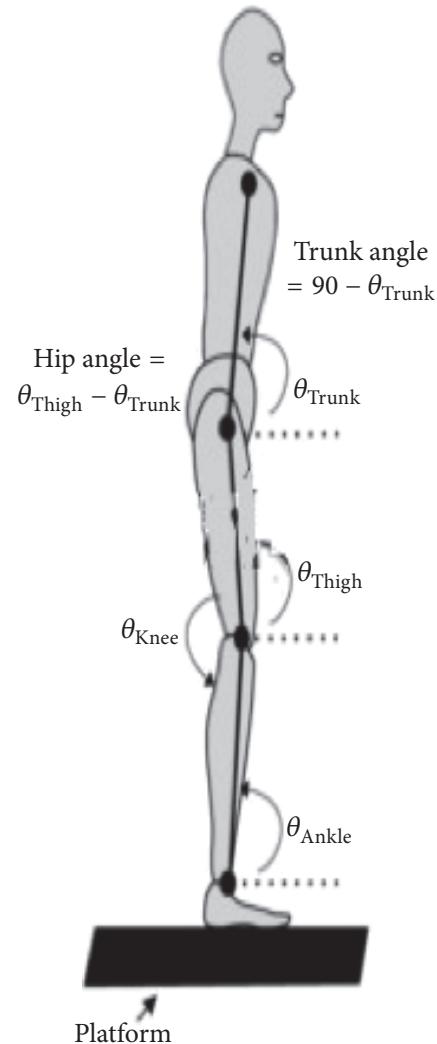

(a)

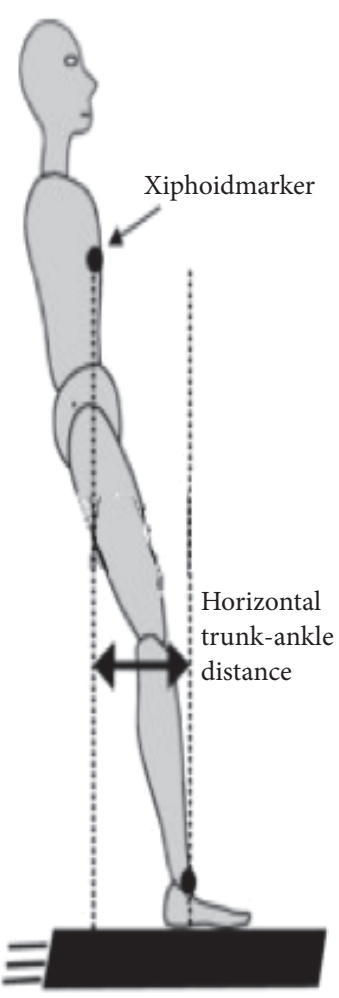

(b)

FIgURE 3: Alternative trunk angle calculation [7].

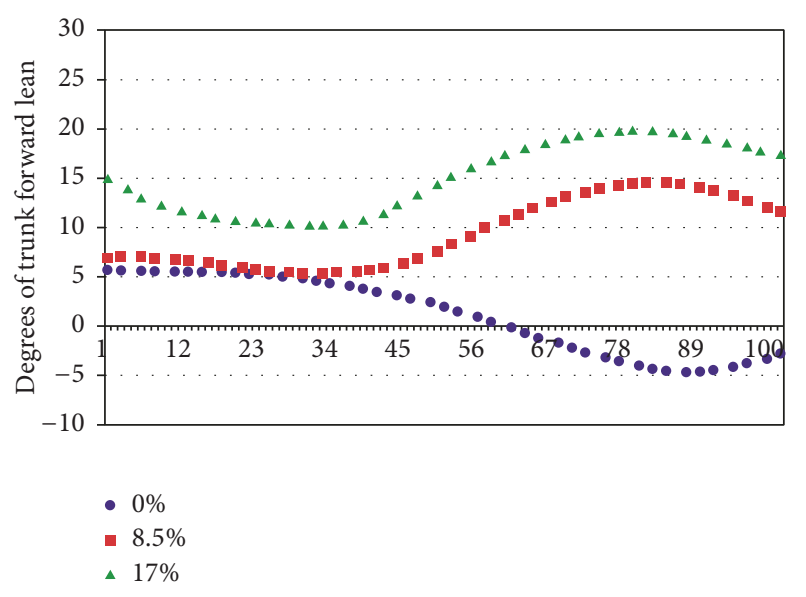

FIGURE 4: Effects of children walking while carrying different backpack load [11].

parameters are crossed; these parameters are sometimes correlated and sometimes uncorrelated [5]. The correlation of lower back pain with some of the parameters is given in Table 2.

From Table 2, the correlation between the mentioned parameters can be calculated for lower back pain.
Pearson's correlation formula is used to calculate the correlation between backpack weight and lumbar lordosis [12]:

The correlation $R$ for lower back pain is found to be 1 . With such strong positive correlation, between the backpack weight and the lumbar bend, it is very clear that the relation of lower back pain with weight of the backpack is very prominent.

Korovessis et al. also concluded [5] that a 5-degree or more trunk angle causes lower back pain in children. Following the Pearson correlation calculation [13], we found the normal ranges of curvature for different parts of the spine and the threshold for bend beyond which it causes aches; these values are shown in Table 3.

Due to the strong correlation with the backpack weight and lower range of curvature, the lumbar spine is chosen to be the subject for the back posture monitoring system. Our prototype uses a simple flex sensor which must be placed in the lower back of the user that leads to measurement of lumber curvature.

\section{Total System Design}

The system in question is a measurement system that measures the lumbar curvature. Therefore, it will require a sensor, a signal conditioning unit, a signal-processing unit, and a 
TABLE 1: Curvature of different parts of spine with different weights of backpacks at different position.

\begin{tabular}{|c|c|c|c|c|}
\hline & Condition & Mean curvature (degree) & Standard deviation & $P$ value \\
\hline \multirow{2}{*}{ Thoracic angle } & $0-10 \%$ body weight [3]. & 47.4 & 13.6 & 0.157 \\
\hline & Body weight $>10 \%[3]$. & 43.8 & 12.7 & 0.157 \\
\hline \multirow{2}{*}{ Lumbar lordosis angle } & $0-10 \%$ body weight [3]. & 26.6 & 14.3 & 0.266 \\
\hline & Body weight $>10 \%[3]$. & 23.9 & 11.7 & 0.266 \\
\hline \multirow{22}{*}{ Trunk flexion } & 0-25\% body weight [9]. & $9.66-3.06$ & $7.87-2.06$ & $<0.001$ \\
\hline & After exercise [10]. & 10.5 & 4.5 & 0.04 \\
\hline & Body weight $<10 \%[8]$. & 4.2 & 1.9 & 0.179 \\
\hline & \multirow{2}{*}{ Stand, body weight = 0\% [11] } & & & 0 \\
\hline & & 0 & 0.25 & \\
\hline & \multirow{2}{*}{ Stand, body weight $<10 \%$ [11]. } & 2.15 & 0.15 & \\
\hline & & 2.4 & 0 & \\
\hline & \multirow{2}{*}{ Stand, body weight $>10 \%$ [11]. } & 6.2 & 0.6 & \\
\hline & & 5 & 0 & \\
\hline & \multirow{2}{*}{ Walk, body weight = 0\% [11]. } & 0.5 & 5.2 & \\
\hline & & 8.4 & 3.6 & \\
\hline & \multirow{2}{*}{ Walk, body weight < 10\% [11]. } & 9.9 & 4.6 & \\
\hline & & 9.75 & 2.05 & \\
\hline & \multirow{2}{*}{ Walk, body weight > 10\% [11]. } & 15 & 4.8 & \\
\hline & & 7.8 & 6.1 & \\
\hline & \multirow{2}{*}{ Run, body weight = 0\% [11]. } & 10.9 & 2.2 & \\
\hline & & 10.6 & 4.7 & \\
\hline & \multirow{3}{*}{ Run, body weight < 10\% [11]. } & 23 & 1.1 & \\
\hline & & 17.15 & 2.88 & \\
\hline & & 21.6 & 3.2 & \\
\hline & \multirow[t]{2}{*}{ Run, body weight > 10\% [11]. } & \multicolumn{2}{|c|}{10.95} & \\
\hline & & 2.65 & & \\
\hline \multirow{2}{*}{ Sacral angle } & Body weight $<10 \%[8]$. & 24.5 & 9.8 & 0.054 \\
\hline & Body weight $>10 \%[8]$. & 19.5 & 10.2 & 0.054 \\
\hline
\end{tabular}

TABLE 2: Correlation between lower back pain and other parameters $[5]$.

\begin{tabular}{lcccc}
\hline Pain type & $\begin{array}{c}\text { Weight of } \\
\text { backpack }\end{array}$ & Thoracic & Lumbar & Scoliosis \\
\hline $\begin{array}{l}\text { Lower } \\
\text { back pain }\end{array}$ & 0.02 & 0.016 & 0.1 & 0.08 \\
$\begin{array}{l}\text { Thoracic } \\
\text { kyphosis }\end{array}$ & 0.16 & -0.035 & 0.01 & 0.009 \\
\hline
\end{tabular}

TABLE 3: Threshold bend for different parts of the spine that is responsible for causing pain.

\begin{tabular}{lcc}
\hline Section of spine & $\begin{array}{c}\text { Range of values } \\
\text { (degree) }\end{array}$ & What causes pain \\
\hline Thoracic [13] & $20-40$ & Lower than 20 \\
Lumbar [13] & $10-20$ & Higher than 20 \\
Trunk flexion [14] & $\begin{array}{c}\text { No significant } \\
\text { (forward relative } \\
\text { to vertical) }\end{array}$ & \begin{tabular}{c} 
correlation \\
\hline
\end{tabular} \\
\hline
\end{tabular}

display device. Keeping these factors in mind the following elements were chosen to be part of the system:

(1) Flex sensor

(2) Arduino

(3) Resistor (for potential division)

(4) Bread board

(5) LED (as a signalling right/wrong posture)

(6) Wires.

The component connection is shown in Figure 5.

In order to have a fully functioning system the methodology flowchart in Figure 6 has been followed.

In Figure 7, the general idea of how the sensor will measure back posture has been shown. As mentioned before, the sensor will be attached to a wearable belt against the back of the user. As the back posture changes, the sensor's angle and therefore its resistance change. As it crosses the threshold angle for good posture, the system will trigger an alarm. 


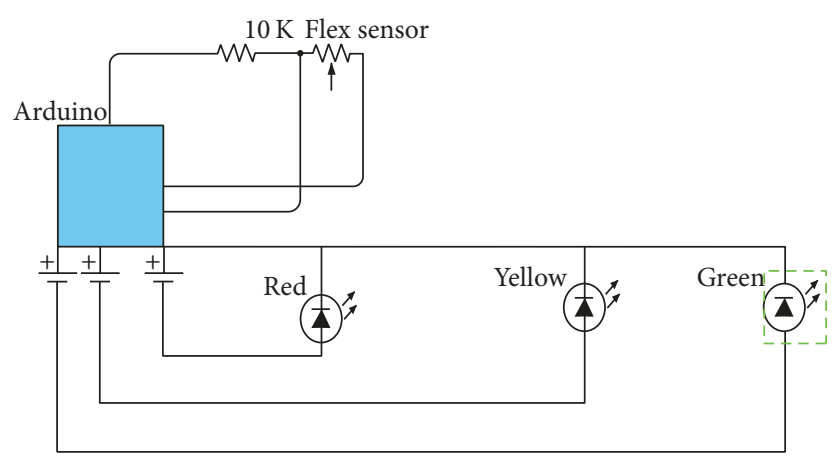

Figure 5: Circuit diagram of the designed system.

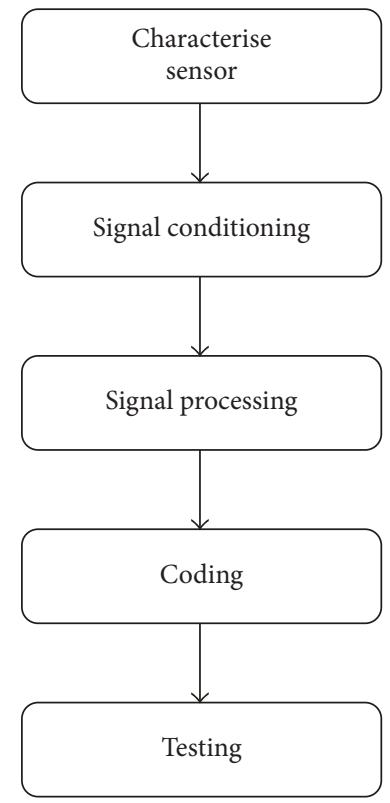

Figure 6: Methodology flowchart.

$$
\mid
$$

(a) Sensor when back is straight

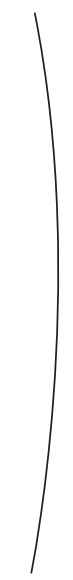

(b) Sensor bends with change in posture

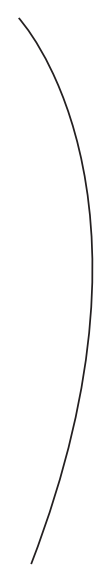

(c) System will trigger alarm as sensor angle crosses the good posture threshold
FIGURE 7: Working principle of the system.
TABLE 4: Relevant flex sensor properties.

\begin{tabular}{lc}
\hline Property & Value \\
\hline Flat resistance & $10 \mathrm{k} \Omega$ \\
Resistance tolerance & $\pm 30 \%$ \\
Height & 0.017 inches \\
Length (active) & 3.750 inches \\
Temperature length & -35 to 80 degrees Celsius \\
Bend resistance & $60 \mathrm{k} \Omega$ to $110 \mathrm{k} \Omega$ \\
\hline
\end{tabular}

3.1. Sensor Characterisation and Modelling. The flex sensor used in this case is from the FS series. The electrical specifications of the sensor are given in Table 4 [15].

The sensor was connected to a multimeter and bent at different angles to find the resistance at particular angles. The angles (bent) were measured using a protractor. This experiment has been carried out numerous times and at different environments; the data found was recorded and tabulated; from this, the sensor's error band was calculated and is shown in Figure 8.

The graph in Figure 8 shows several thin lines that account for individual tests and the bold line that accounts for the average value of all the tests; this leads the relationship for resistance versus angle to be

$$
R=0.0053 A 2-0.026 x+12,
$$

where $R$ is resistance and $A$ is angle, and the average standard deviation was thus found:

$$
H=1.025 \text {. }
$$

Additionally the flat resistance of the sensor was found to be $13 \mathrm{k} \Omega$, which is $3 \mathrm{k} \Omega$ higher than that of the sensor specifications.

The modelling of the flex sensor shows that it follows specific patterns for change in resistance; the deviations that occurred due to different environment are minor and can be neglected.

3.2. Signal Conditioning. Signal conditioning is necessary to present the sensor's results in a form that is understandable. The following forms of signal conditioning were used for the posture monitoring system.

(1) Power Supply. In order to develop a measurement system we need a power supply that is suitable for all the components. Similarly since the measurement itself is not carried out in form of electrical energy, a transducer is required for electrically quantifying other forms of measurements, for the hardware's benefit. Therefore a $5 \mathrm{~V}$ power supply is applied across the flex sensor, which in turn due to change in resistance shows a change in voltage.

(2) Potential Divider. The entirety of the power supply cannot be applied across the sensor as in that case the voltage drop across the flex sensor will be the same as the supplied power regardless of any change in resistance. This requires the need of a potential divider, which will give a nice voltage drop 


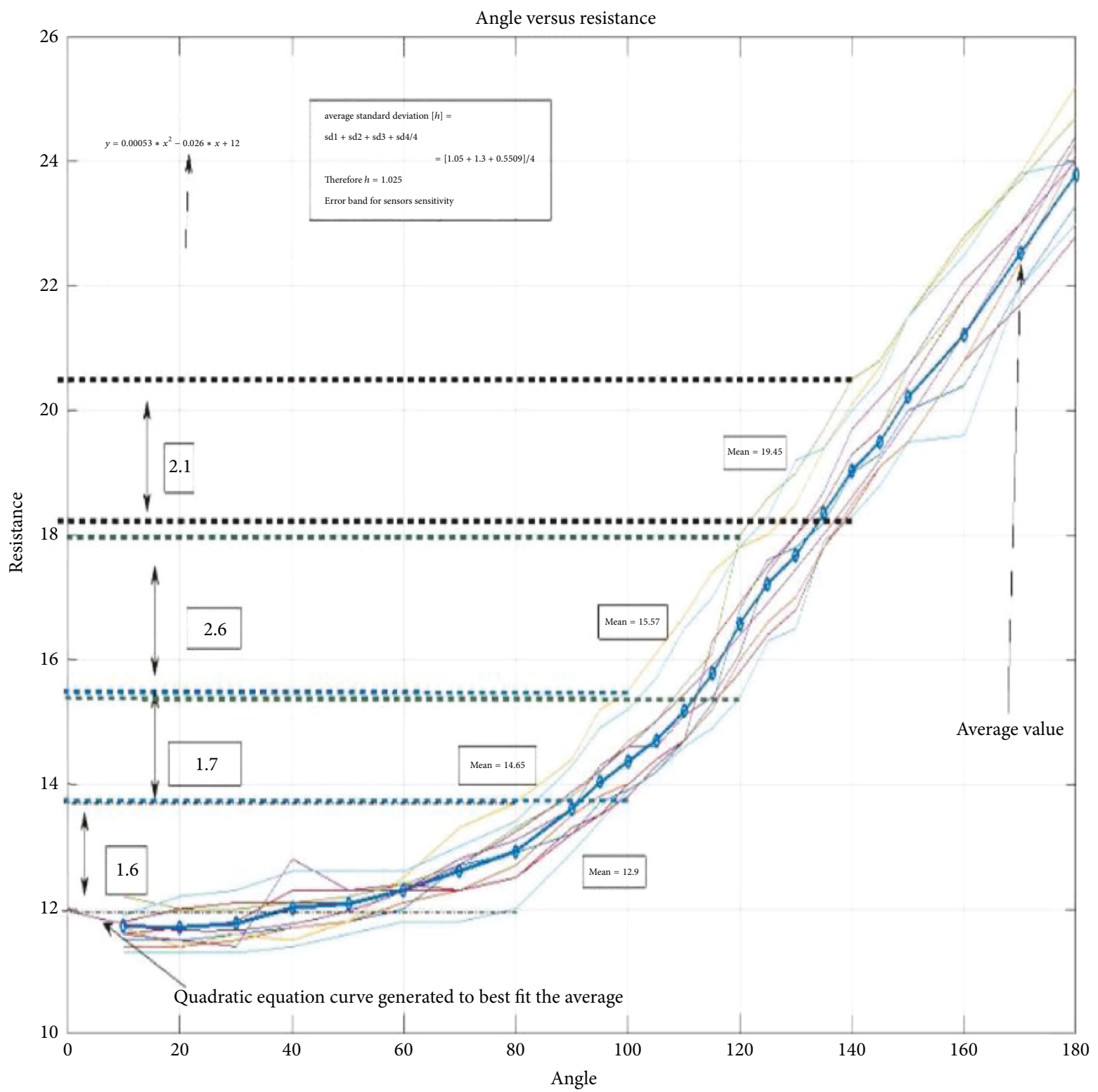

FIGURE 8: Flex sensor resistance against angle bent [16].

across the flex sensor and will have a good range to work with in accordance with the resistance range of the flex sensor.

In order to find the ideal potential divider for the system, two resistors of $10 \mathrm{~K} \Omega$ and $2 \mathrm{~K} \Omega$ were used. A voltmeter was connected in parallel with the flex sensor and the voltage drop across it was measured and tabulated with the change in flex sensor angle. After a number of experiments with both resistors, the average values were taken and plotted, shown in Figure 9.

Figure 9 shows that the $10 \mathrm{~K} \Omega$ potential divider has a drop that ranges between $2.9 \mathrm{~V}$ and $3.4 \mathrm{~V}$. On the other hand the $2 \mathrm{~K} \Omega$ potential divider has a voltage drop that ranges between $4.3 \mathrm{~V}$ and $4.5 \mathrm{~V}$ giving a working range of just $0.2 \mathrm{~V}$ where the $10 \mathrm{~K} \Omega$ resistor gives a larger working range of $0.5 \mathrm{~V}$. This is the reason why a $10 \mathrm{~K} \Omega$ resistor was chosen as the potential divider for the back posture monitoring system. Larger resistor would have a smaller voltage drop and would require amplification. Using any signal-amplifying unit was avoided to reduce system complexity.

3.3. Signal Processing. Signal processing for the back posture monitoring system was done using hardware and software. Hardware for signal processing, that is, the Arduino, carries all the signal-processing tasks. It executes all the instructed commands such as picking up data from input, analogue to 


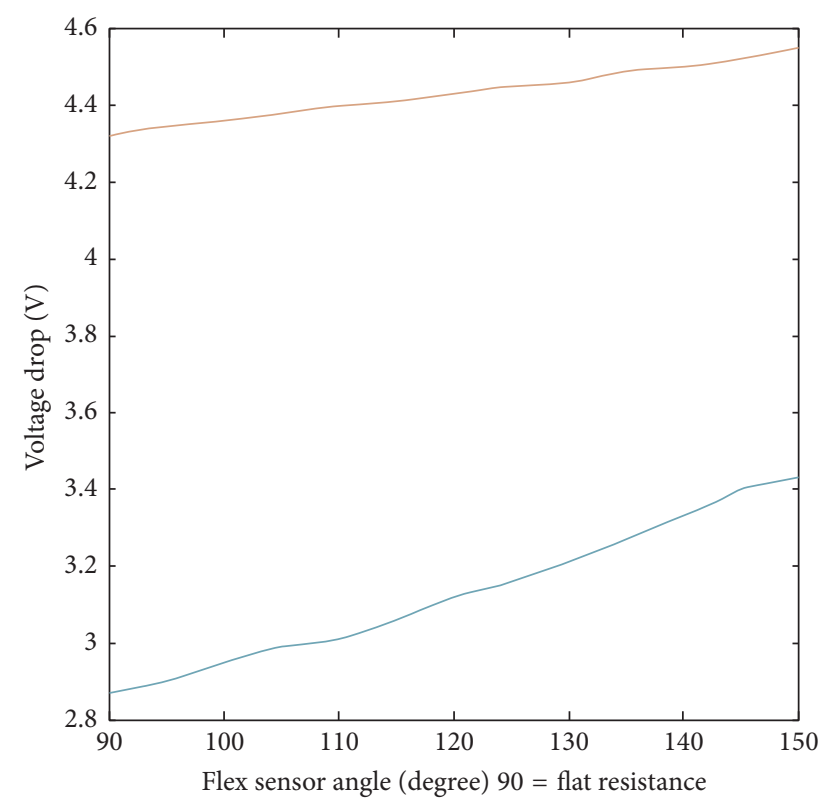

FIGURE 9: Voltage drop measurement.

TABLE 5: Arduino Uno technical specifications.

\begin{tabular}{lc}
\hline Microcontroller & atmega328p \\
\hline Operating voltage & $5 \mathrm{v}$ \\
Input voltage & $7-12 \mathrm{v}$ \\
(recommended) & $6-20 \mathrm{v}$ \\
Input voltage (limit) & 14 (of which 6 provide PWM output) \\
Digital i/o pins & 6 \\
PWM digital i/o pins & 6 \\
Analogue input pins & 20 ma \\
Dc current per i/o pin & 50 ma \\
Dc current for 3.3 v pin & used by bootloader \\
Flash memory & $2 \mathrm{~kb}$ (atmega328p) \\
SRAM & $1 \mathrm{~kb}$ (atmega328p) \\
EEPROM & $16 \mathrm{MHz}$ \\
Clock speed & $68.6 \mathrm{~mm}$ \\
Length & $53.4 \mathrm{~mm}$ \\
Width & $25 \mathrm{~g}$ \\
Weight &
\end{tabular}

digital conversion, filtering, digital to analogue conversion, and delaying and then sending the data to output. These instructions were programmed to the Arduino using the software "Arduino" and by the use of C programming language.

The technical specifications of the Arduino Uno used in this project are given in Table 5 [17].

As the electrical signal from the flex sensor is coming in analogue form, it is necessary to convert it into digital to perform digital filtering and display the result in a computer. Here the $A / D$ conversion takes place in a range of 0 to $5 \mathrm{v}$

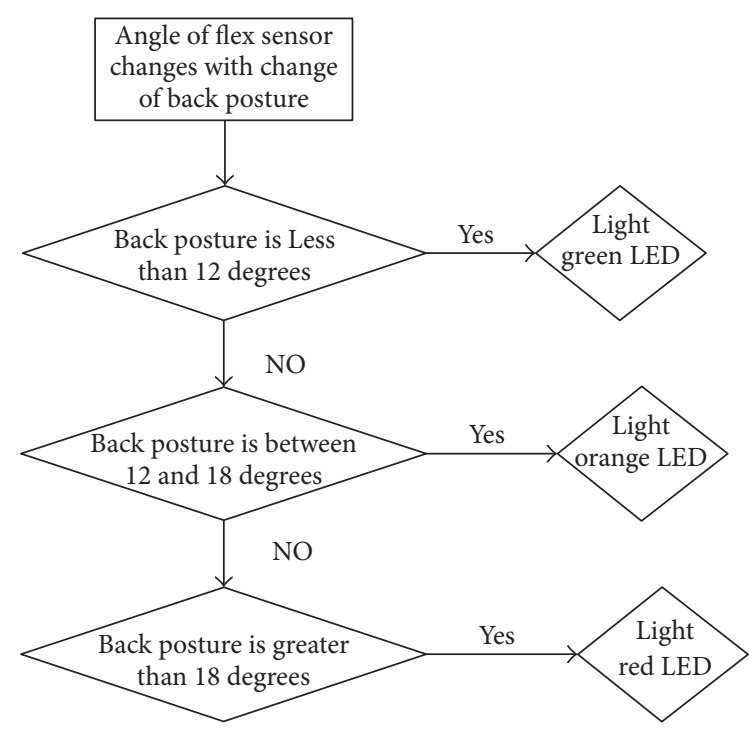

FIGURE 10: Monitoring methodology.

in analogue domain and 0 to 1023 in digital domain with the help of the built-in ADC in the Arduino Uno board [18].

To trigger external alarms and displays such as LED, speaker, buzzer, seven-segment display, or LCD display, the digital signal needs to be converted back to analogue. The $\mathrm{D} / \mathrm{A}$ conversion is carried out with a resolution of 8 bits; therefore the output is mapped in the range of 0 to 255 where 0 corresponds to $0 \mathrm{v}$ and 255 corresponds to $5 \mathrm{v}$ [19].

Despite filtering being a conditioning aspect, the hardware is programmed to carry out this task since it is easily carried out in the digital domain thereby easing the complexity of the analogue domain of the system. The type of digital filter used in this system is a moving mean, which averages the values of 3 consecutive measurements and sends it as output, thereby removing large fluctuations.

3.4. Type of Display. One of the most important aspects of a measurement system is to be able to present the result in a simple manner so that the user can understand it without difficulty; the back posture monitoring system's result displaying aspect has been designed keeping that in mind.

15 experiments were conducted where the digital output from the Arduino displayed in computer monitor using serial port was tabulated against different angles of the flex sensor. According to the research addressed in the previous section of this report, if lumbar part of the back is bent less than 15 degrees, it is deemed as good posture. Anything above 20 degrees is considered as bad posture, which will cause lower back pain. The average of the digital output at 15 degrees was set as the threshold for good posture, the average range of outputs between 15 and 18 degrees was set to be marginally bad posture, and the average digital output found at 18 degrees was set as the threshold for bad posture using $\mathrm{C}$ coding.

Figure 10 shows the general methodology of monitoring back posture of the user. The system is designed to have simple input and output system, since it is for the use of children. There are three LEDs of different colours that will 
Normal Posture (0-12 Degree From Flat Position)

Normal Posture (0-12 Degree From Flat Position)

Bad Posture (More than 18 Degree From Flat Position)

Bad Posture (More than 18 Degree From Flat Position)

Normal Posture (0-12 Degree From Flat Position)

Normal Posture (0-12 Degree From Flat Position)

Normal Posture (0-12 Degree From Flat Position)

Box 1: Screenshot of serial monitor output.

TABLE 6: Correlation between height (in range) and spinal length.

\begin{tabular}{lc}
\hline Height range & Correlation \\
\hline $152 \mathrm{~cm}$ or less & 0.29 \\
$153-161 \mathrm{~cm}$ & 0.3 \\
$162 \mathrm{~cm}$ or greater & 0.31 \\
\hline
\end{tabular}

help in identification of current posture. The green LED is on when the posture output is less than the threshold set using $\mathrm{C}$ coding, orange LED will light up when output is within the range of 12-18 degrees, and red LED will light up when the output exceeds the threshold set for 18 degrees.

The user can also monitor back posture using a computer monitor where the serial monitor of the computer will constantly update the back posture status with these messages:

(i) Good posture (less than 12 degrees)

(ii) Marginally bad posture (between 12 and 18 degrees)

(iii) Bad posture (greater than 18 degrees).

Box 1 shows how the system will update the user of their posture in the serial monitor of a PC.

3.5. Determining the Position of the Sensor. One concern about the position of the system in a child's body, the part of the torso it must be placed upon, was solved by a paper written by Lin et al. [20]; here it was stated that there is a correlation of human height and the length of each of their spinal vertebrae. From this relationship, the perfect position of fitting the belt (that was in turn fitted with the sensor) was found. Correlations mentioned in a paper written by Lin et al. are given in Table 6 with examples for how to calculate the ideal position to wear the belt [20].

Lin et al. state how the relationship for body height and spine length from sacral to thoracic is 0.31 and thus the following calculation can be made [20]:

$$
\begin{aligned}
& \text { Human height }=x \\
& \text { Spine height }=0.31 * x \\
& \text { Number of thoracic columns, } t=12 \\
& \text { Number of lumbar columns, } l=5 \\
& \text { Number of sacral columns, } s=5 .
\end{aligned}
$$

Therefore, the right place to wear the sensor above tailbone is given by

$$
\text { Position of the sensor }=s * 0.31 * x \text {. }
$$

TABLE 7: Results for accuracy.

\begin{tabular}{lccc}
\hline \multicolumn{3}{c}{ Incident occurrence number of exp. percentage } \\
\hline False negative & 8 times & 240 occasions & $3.30 \%$ \\
False positive & 5 times & 240 occasions & $2.08 \%$ \\
\hline
\end{tabular}

For example, for a $164 \mathrm{~cm}$ human being, that is, $5^{\prime} 4$ approximately, correlation $=0.31[21]$.

The spine height from (thoracic to sacral) $=50 \mathrm{~cm}$

Each vertebrae length $=50 / 22(22 \mathrm{pc}, 12$ thoracic, 5 lumbar, and 5 sacral $)=2.3 \mathrm{~cm}$

Sacral column length $=11.5$.

Therefor the belt must be placed $11.5 \mathrm{~cm}$ above the tailbone.

3.6. Testing the System. After taking multiple measurements for the system, the digital output form the serial monitor was against the input angle of the flex sensor and was plotted to find the error band and shown in Figure 11.

Here the blue line represents the ideal value and the yellow and red lines show the error maximum and minimum limit; " $h$ " was determined to be 1.048 .

The completed system was worn by two different people for testing. 240 instances were checked for positive, negative, false positive, and false negative values, that is, false negative: when an expected green is shown orange or when an expected orange is shown red, and false positive: when an expected red shows an orange or an expected orange shows a green.

Table 7 shows the number of times false positive or negative was found true for the system.

Therefor the accuracy of the system stands to be

$$
\begin{aligned}
& E=100 \%-(\text { false negative }+ \text { false positive }) \%, \\
& E=100 \%-(3.3 \%+2.08 \%), \\
& E=94.62 \% .
\end{aligned}
$$

\section{Limitation}

There are several limitations of the developed back posture monitoring system.

(1) The flex sensor used for the system is not the best available in the market. It is more prone to errors. It is affected by temperature and the output of the sensor 


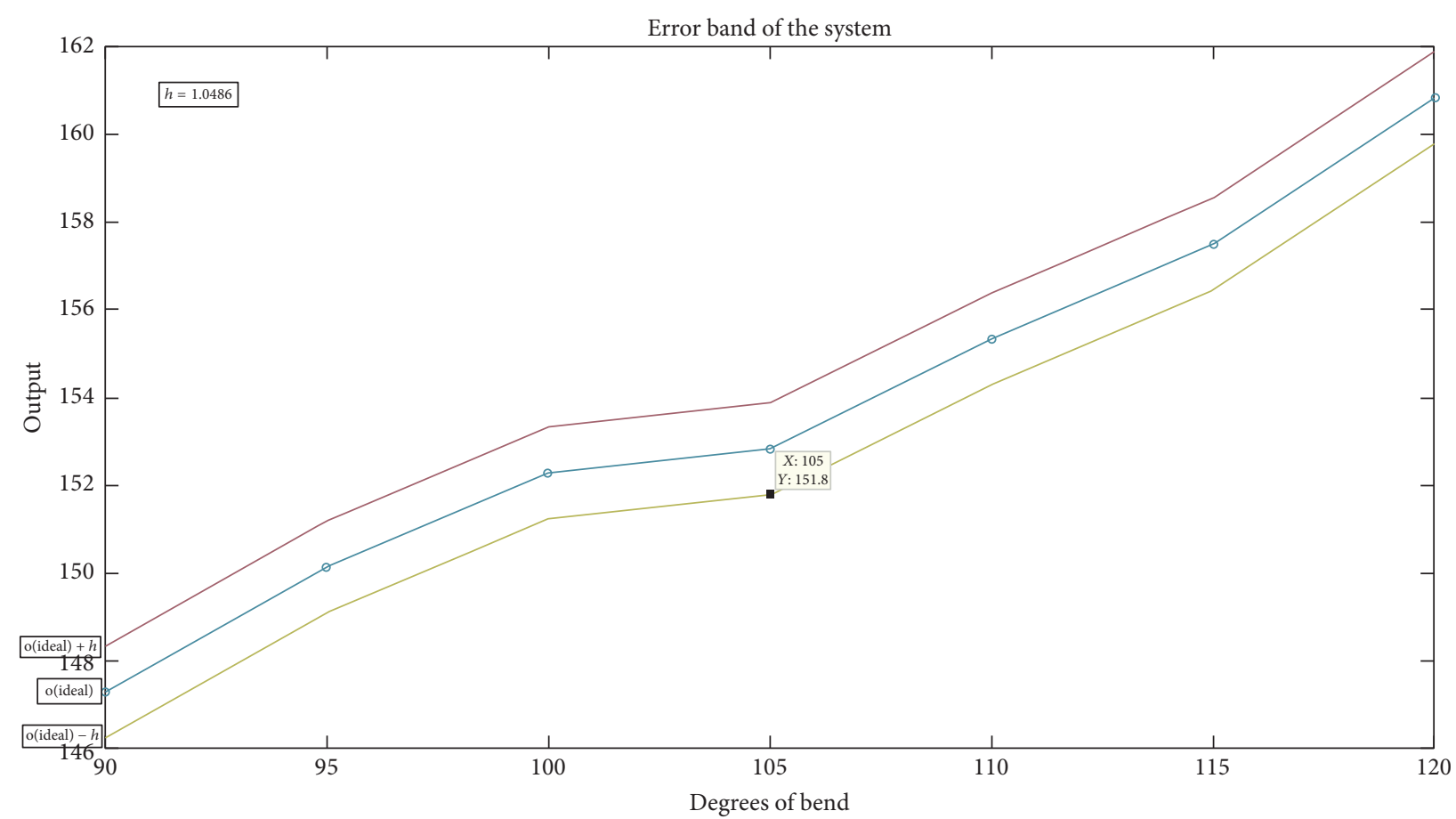

FIGURE 11: Error band of the system represented by three different lines. The blue is the ideal value; the yellow and red are ideal $+h$ and ideal $-h$, respectively [16].

becomes unpredictable with increasing temperature (no correlation could be found with increasing temperature and resistance).

(2) The system is dependent on computer, via the USB cable, rendering the system immobile.

(3) The accuracy of the system was calculated based on only two users, thus making the system susceptible to unknown errors.

(4) The developed system only has optical alarm (i.e., LEDs). User with visual impairment will not be able to notice whether the posture is good or bad.

(5) The system lacks a data-recoding feature.

(6) Reliability of the system is not very high due to the fact that if the user has a fever, the resistance of the sensor will be affected by it; additionally, since the system depends on only one sensor, the lack of redundancy and diversity is a significant issue.

\section{Future Prospects and Improvements}

Improvements can be made by the use of

(1) better sensor, impervious to temperature and better sensitivity to angle displacement,

(2) an external battery to power the Arduino, making it portable,

(3) a smaller board like Arduino LilyPad which can make the overall system more portable,
(4) using multiple sensors with diversity which will get rid of common-mode failures and make it more reliable,

(5) testing the system on more users which could produce a more reliable accuracy result of the system, thus making it resistant to unknown problems,

(6) a memory (USB/flash) used for storing previous records which could have been helpful for parents to monitor their children's posture a for an entire day or week; this can be simply done by the use of Arduino Ethernet shield instead of an Arduino Uno or using SparkFun shield or separately implanting a SD card slot into the Arduino Uno [21],

(7) using a wireless device which may allow the user to monitor their posture from their phones,

(8) an additional sound alarm which may be helpful for the visually impaired.

\section{Conclusion}

Today's youth is subjected to heavy weighted backpack; this system allows the user to be constantly reminded of the condition of their posture. The care of which is essential since early age lower back pain persists while growing and thus can become chronic later in life. Since the weight of heavy backpacks on children can cause severe deterioration on the lumbar part of the spine, monitoring is essential by either parents or, by the help of this system, children themselves, enabling them to decide on making their backpacks lighter, essentially relieving their spines of the daily burden and 
ensuring better posture later in life. The developed back posture monitoring system follows all the set criteria that a standard measurement system should have such as proper signal conditioning, processing, accuracy, cost effectiveness, and simplicity in design and it presents the required result in an understandable manner. The reliability of the back posture monitoring system can be improved by making it suitable for any environment and by adding redundancy and diversity. This simple device could be proven very significant in ensuring that the future generation of the world can grow up without lingering issues of lower back pain and permanent bad posture.

\section{Conflicts of Interest}

The authors declare that there are no conflicts of interest regarding the publication of this paper.

\section{References}

[1] K. C. Wong, R. Y. Lee, and S. S. Yeung, "The association between back pain and trunk posture of workers in a special school for the severe handicaps," BMC Musculoskeletal Disorders, vol. 10, article 43, 2009.

[2] S. Basaran and I. C. Benlidayi, "Comparative study of lumbosacral alignment in elderly versus young adults: data on patients with low back pain," Aging Clinical and Experimental Research, vol. 27, no. 3, pp. 297-302, 2014.

[3] K. Walicka-Cupryś, R. Skalska-Izdebska, M. Rachwał, and A. Truszczyńska, "Influence of the weight of a school backpack on spinal curvature in the sagittal plane of seven-year-old children," BioMed Research International, vol. 2015, Article ID 817913, 6 pages, 2015.

[4] M. J. Moore, G. L. White, and D. L. Moore, "Association of relative backpack weight with reported pain, pain sites, medical utilization, and lost school time in children and adolescents," Journal of School Health, vol. 77, no. 5, pp. 232-239, 2007.

[5] P. Korovessis, G. Koureas, and Z. Papazisis, "Correlation between backpack weight and way of carrying, sagittal and frontal spinal curvatures, athletic activity, and dorsal and low back pain in schoolchildren and adolescents," Journal of Spinal Disorders and Techniques, vol. 17, no. 1, pp. 33-40, 2004.

[6] J. M. Lambrecht, M. L. Audu, R. J. Triolo, and R. F. Kirsch, "Musculoskeletal model of trunk and hips for development of seatedposture-control neuroprosthesis," The Journal of Rehabilitation Research and Development, vol. 46, no. 4, pp. 515-528, 2009.

[7] Wordpress, "Pain behind knee sitting too long," Hip Angle, 2016.

[8] D. Czaprowski, P. Pawłowska, Ł. Stoliński, and T. Kotwicki, "Active self-correction of back posture in children instructed with 'straighten your back' command," Manual Therapy, vol. 19, no. 5, pp. 392-398, 2014.

[9] M. Ramprasad, J. Alias, and A. K. Raghuveer, "Effect of backpack weight on postural angles in preadolescent children," Indian Pediatrics, vol. 47, no. 7, pp. 575-580, 2010.

[10] J. M. Hart, D. C. Kerrigan, J. M. Fritz, and C. D. Ingersoll, "Jogging kinematics after lumbar paraspinal muscle fatigue," Journal of Athletic Training, vol. 44, no. 5, pp. 475-481, 2009.

[11] S. Goodgold, K. Mohr, A. Samant, T. Burns, T. Park, and L. Gardner, "Trunk forward lean during backpack usage under varying load and task demands: pilot findings of two boys," Pediatric Physical Therapy, vol. 13, no. 4, p. 196, 2001.
[12] T. de Jonge, J. F. Dubousset, and T. Illés, "Sagittal plane correction in idiopathic scoliosis," Spine, vol. 27, no. 7, pp. 754760, 2002.

[13] Pearson Correlation Coefficient Calculator, http://www.socscistatistics.com/.

[14] T. Kienbacher, E. Fehrmann, R. Habenicht et al., "Age and gender related neuromuscular pattern during trunk flexionextension in chronic low back pain patients," Journal of NeuroEngineering and Rehabilitation, vol. 13, no. 1, article 16, 2016.

[15] Spectra Symbol, FlexSensor FS Specification, spectrasymbol.com, 2016.

[16] C. Moler, MATLAB, MathWorks, 2016.

[17] Arduino, "Arduino ArduinoBoardUno," 2016, https://www.arduino.cc/en/main/arduinoBoardUno.

[18] Arduino.cc, “Arduino AnalogRead," https://www.arduino.cc/en/ Reference/AnalogRead.

[19] Arduino.cc, "Arduino-AnalogWrite," 2016, https://www.arduino.cc/en/Reference/AnalogWrite.

[20] I. S. Lin, C. L. Lee, J. L. Wu, C. F. Wang, C. G. Liu, and Y. T. Lee, "Comparison of body height and vertebral column length in Chinese parturients," Anaesthesiologica Sinica, vol. 30, no. 1, pp. 21-25, 1992.

[21] Arduino.cc, "Arduino SDCardNotes," 2016, https://www.arduino.cc/en/Reference/SDCardNotes. 


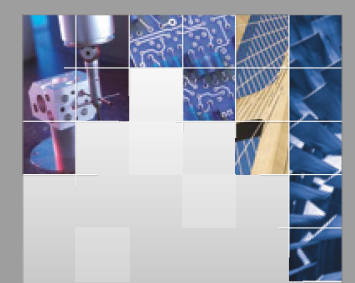

\section{Enfincering}
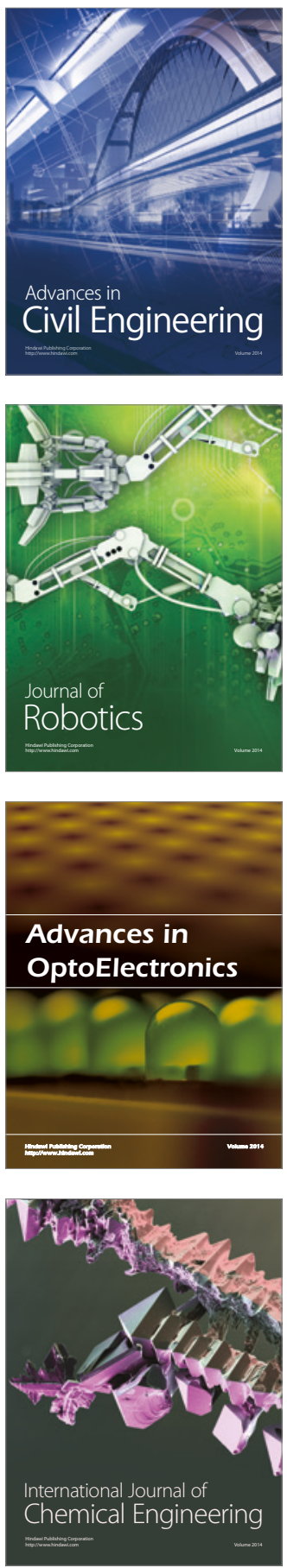

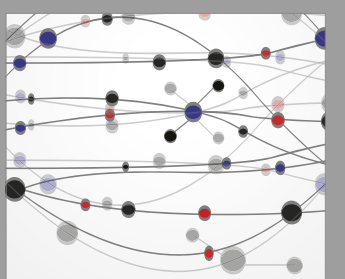

The Scientific World Journal

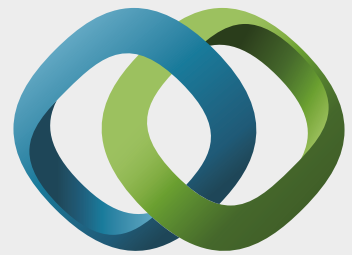

\section{Hindawi}

Submit your manuscripts at

https://www.hindawi.com
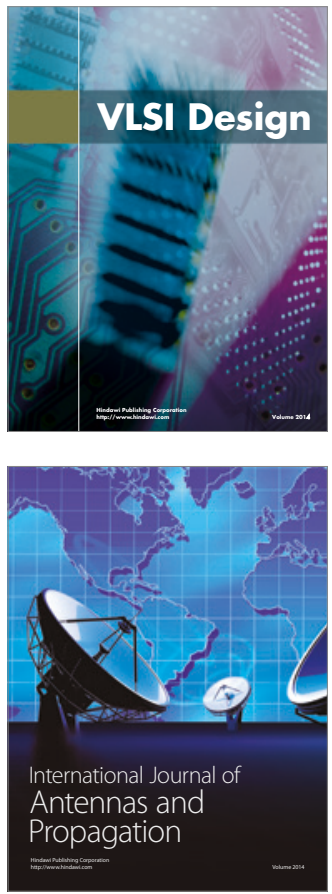

\section{Rotating}

Machinery
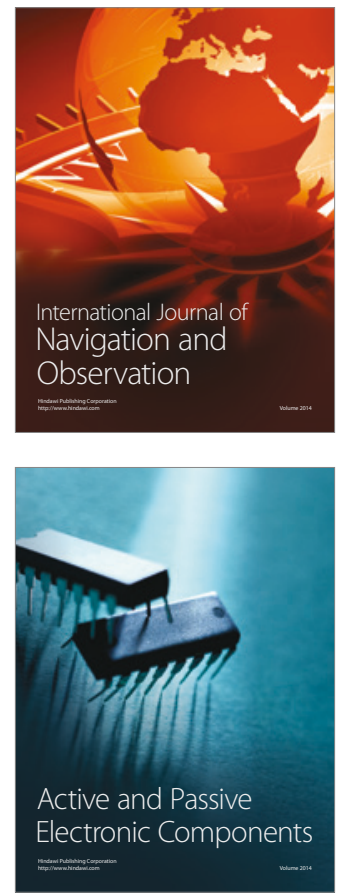
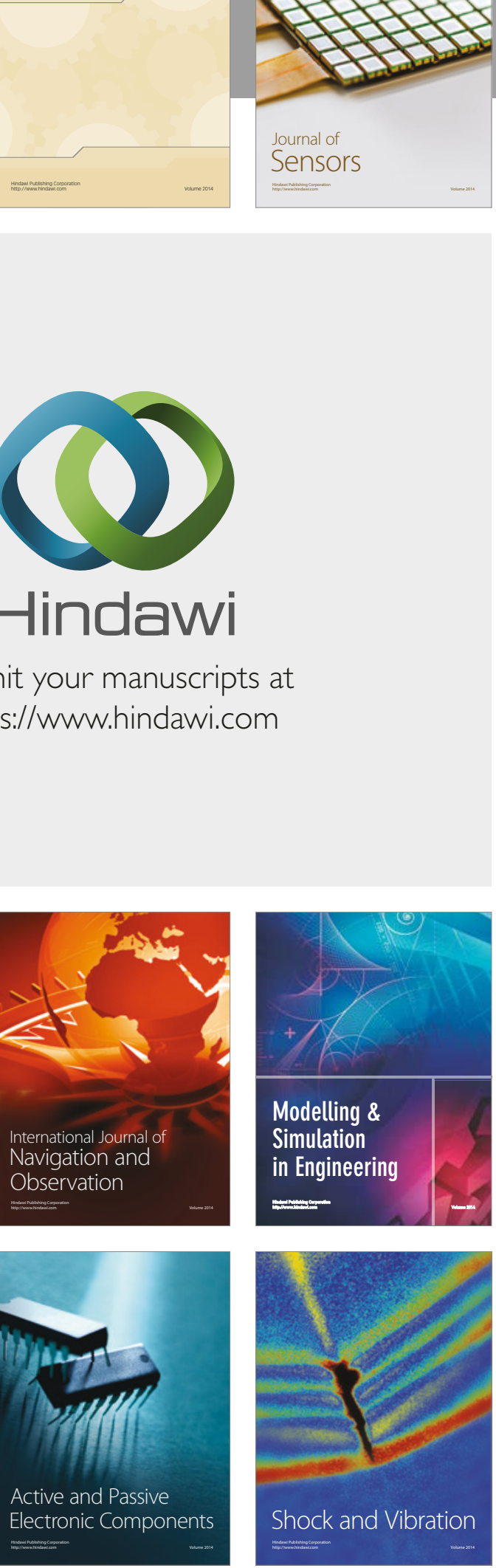
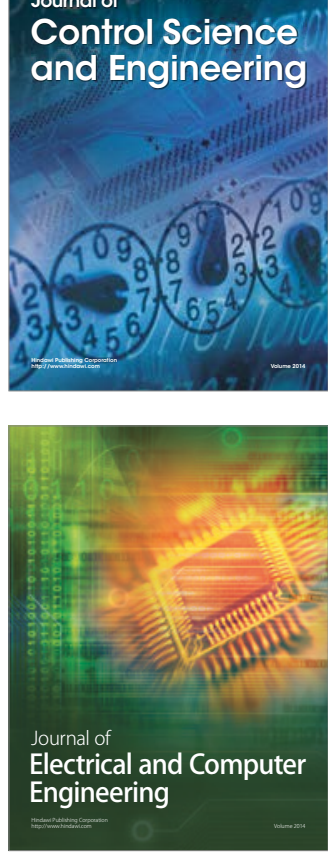

Distributed

Journal of

Control Science

and Engineering
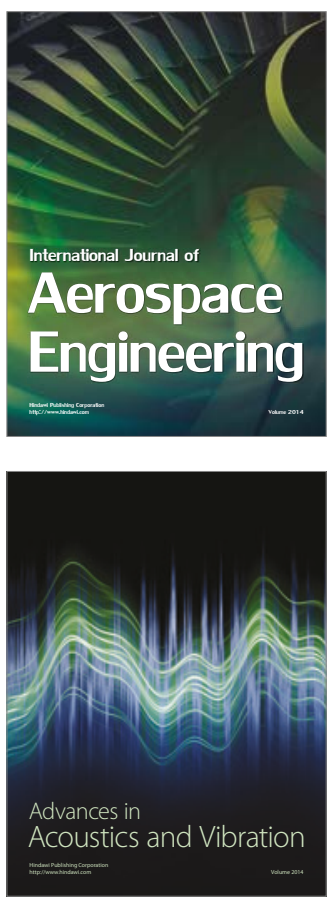

Sensor Networks 